\title{
INVESTIGATING THE USE OF REAL-TIME DATA IN NUDGING PATIENTS' EMERGENCY DEPARTMENT (ED) ATTENDANCE BEHAVIOUR
}

\author{
Navonil Mustafee \\ University of Exeter Business School \\ Streatham Campus \\ Exeter EX4 4ST, Devon, UK \\ N.Mustafee@exeter.ac.uk \\ Susan Martin \\ Horizon Centre, Torbay Hospital \\ Torbay \& South Devon NHS Foundation Trust \\ Torquay TQ2 7AA, UK \\ Susan.Martin@nhs.net
}

\author{
John H. Powell \\ University of Exeter Business School \\ Streatham Campus \\ Exeter EX4 4ST, Devon, UK \\ J.H.Powell@exeter.ac.uk
}

\author{
Andrew Fordyce \\ Horizon Centre, Torbay Hospital \\ Torbay \& South Devon NHS Foundation Trust \\ Torquay TQ2 7AA, UK \\ Andrew.Fordyce@nhs.net
}

\author{
Alison Harper \\ University of Exeter Business School \\ Streatham Campus \\ Exeter EX4 4ST, Devon, UK \\ AH596@exeter.ac.uk
}

\begin{abstract}
Decision-making in healthcare is a complex process involving multiple stakeholders. One such stakeholder category is the intended users of the system itself - the patients. We present a study in which users use real-time hospital operations data to make attendance choices. The work was carried out with the Torbay \& South Devon NHS Foundation Trust (TSDFT) and its network of Minor Injury Units (MIUs) and one Emergency Department (ED). The aim of this research was to provide information transparency on ED/MIU waiting times which would allow recipients, including, significantly, patients who are in need of urgent medical attention, to make informed decisions as to the facility that could best serve their needs. This work will contribute towards reducing pressure in ED by redistributing demand for minor ailments among the MIUs, since the MIUs have facilities for the treatment of minor injuries and the ED exists mainly for emergency and life-threating conditions.
\end{abstract}

Keywords: Real-time Data, Business Intelligence, Healthcare Analytics, Accident and Emergency, UK National Health Service (NHS), Complexity in Decision Making

\section{INTRODUCTION}

Healthcare can be considered a complex adaptive system (Paley and Eva 2011) and managing these selforganizing systems and designing relevant decision-making processes presents challenges. This complexity increases where there are rapid internal and external changes, and organizations must adapt to these dynamics if they are to survive. In the UK currently, the National Health Service (NHS) is going through a period of transition where, in the period of increasing demand on NHS services, the Trusts are being asked to make drastic cost savings and to manage their budgets even more robustly. Healthcare organizational structures designed for more stable situations are increasingly becoming obsolete. Flexible 
solutions are thus required, and knowledge management can play an increasingly important role in flexible decision-making (Yurtseven and Buchanan, 2016).

The NHS five year forward view set out a vision for the future with the threefold objective of meeting the changing needs of patients, to realize efficiency savings, and to capitalize on the advances in treatment and healthcare technologies. To support these objectives several major new models of care have been proposed which can be implemented locally across England (NHS England, 2015). One care model is on urgent and emergency care and it encourages NHSTrusts to redesign urgent and emergency care for all affected age groups accessing ED. Our research focuses on the urgent and emergency care model. The Keogh review of urgent and emergency care (Urgent and Emergency Care Review Team, 2013) articulates a vision where people with urgent but non-life threatening needs are treated outside of hospitals by services that deliver care in or as close to people's homes as possible; those with more serious or life threatening emergency needs are treated in centers with the very best expertise and facilities. Getting the first part of the vision right will relieve pressure on the hospital based emergency services, thus freeing up resources which could be used to realize the second part of the vision. This vision has been adopted in Torbay \& South Devon (T\&SD) by TSDFT through their urgent care strategy for 2014/15 - 2019/20 (South Devon \& Torbay CCG, 2015). Towards realization of this vision, we have worked with the Trust to develop a healthcare Business Intelligence (BI) application with objective of reducing pressure in Torbay Hospital's ED.

UK NHS has traditionally relied on historical reporting and have focused on reactive rather than proactive analysis and decision making; whilst the former analysis is helpful, it can be argued that it fails to ensure that the business functions can address issues before they happen (Shadi and Mustafee, 2013). BI applications provide a new generation of reporting tools for the NHS since they enable the stakeholders to receive real-time information of "what is happening?" and react to the same. Such information could also ensure that the NHS business functions can address issues before they happen. This is made possible through use of the underlying technologies like online analytical processing (OLAP), real-time visualization (e.g., using BI dashboards to display metrics associated with Key Performance Indicators), web-based platform for reporting, etc. which are present in most BI applications in market today, e.g., Oracle ${ }^{\circledR}$ Business Intelligence, IBM Cognos ${ }^{\circledR}$ and QlikView ${ }^{\circledR}$.

$\mathrm{BI}$ can be defined as the transformation of raw data into meaningful information with the objective of providing effective strategic, tactical and operational insights to decision-making (Evelson 2010). This definition is consistent with the existing literature that focuses predominantly on industrial organizations and how the management could make effective use of BI. However, to place this in healthcare context, it is important that we recognize that, unlike most other sectors that have a well-defined group of customers and products, healthcare includes both external actors (e.g., patients, contractors) and those that are internal, like clinicians, financial controllers and other management functions (Mettler and Vimarlund 2009). Although the former do not have a direct role in the operation of the organization, the decisions they take usually have a bearing on organizational performance. For example, when confronted with the need for urgent and emergency treatment, the intended users (the patients) have to make location decisions as to the place of treatment. If they are unaware of the availability of urgent care services appropriate to meet their needs close to where they are located, they will usually choose to go to ED as they are confident they will be seen and have their needs met. In this paper we investigate the use of existing healthcare operations' data and how it could inform the public about available health and care services in real-time in order that they can access urgent care services where it is more appropriate, thereby leading to a reduction in inappropriate attendance in ED. The availability of real-time information serves to empower the users and increase their levels of self-activation (taking control) in early reduction in anxiety; this also leads to a reduction in 'failure demand'.

The remainder of the paper is organized as follows. Section 2 reviews literature ... Section 3 provides the context of the study. This is followed by the description of the BI application. Section 5 then presents usage statistics, with Section 6 discussing future work and bringing the paper to a close. 


\section{COMPLEXITY IN HEALTHCARE DECISION MAKING - A CASE FOR BI APPLICATIONS}

Decision-making can be a complex process involving multiple stakeholders with varying perspectives and mindsets; and the problem situation must be viewed in the context of the whole system. Intelligent technology can help to overcome some of these difficulties by facilitating learning through knowledge management (Ngai et al. 2014), particularly where effective decisions need to be made quickly. BI can provide accurate, real-time information and valuable support for rapid decision-making. Yurtseven and Buchanan (2016) argued that intelligent knowledge management and decision-support systems provide the opportunity to design self-organizing and self-regulating decision-making systems. Complex systems such as healthcare are large, with multiple subsystems and complex relationships between system components. Ivanov, Sokolov and Kaeschel (2010) remind us that this complexity makes cause-effect relationships difficult to discern. As a result, poor decisions may result in unintended outcomes either immediately, or in the long-term. This can then move the system to unwanted states if decisionmechanisms are not well-designed, particularly in self-adaptive organizations which evolve over time (Yurtseven \& Buchanan, 2016). However healthcare BI applications focus on supporting information evidence-based decision-making by healthcare service providers and managers (Spruit, Vroon \& Batenburg 2014; Simpao et al. 2014; Kao et al. 2016), while patient decisions tend not to be considered.

Kluth et al. (2014) classified approaches to complex decision-making, with one approach being 'focusing on individual factors'. This involves dividing the main complex problem into single smaller problems. This is an appropriate perspective for investigating processes associated with emergency demand, where multiple independent factors within the wider healthcare system contribute to delays for patients and the organization with respect to the 4-hour waiting-time target. These include internal and external healthcare factors such as capacity constraints and lack of availability of GPs; uncontrollable factors such as the weather and demographic variables; and patient-specific factors such as proximity to the emergency department, habitual behavior and lack of knowledge of alternatives. We cannot expect to know complex systems completely, and selecting system boundaries and scope are of vital importance in defining the problem area and the design of decision-support approaches (Daellenbach et al. 2012). Patients are the intended users of the system. Delineating the patient-specific factors and designing a decision-support tool that allows the decision to remain with the patient can investigate the effect of these patient-specific factors on the system. The problem situation must be viewed within its whole-system context, and crucially should be defined with the involvement of a range of stakeholders to resolve plurality of perceptions and objectives. The objective is to improve system equilibrium by facilitating patient self-organizing behavior (Paley and Eva 2011) through a real-time BI application that aims to shift user decisions for emergency care where appropriate.

It has been argued that health IT typically focuses on improving the performance of isolated tasks, rather than on supporting value-added processes of care (Walker and Carayon 2009). The distinction is made between a task-focus and a process-focus, where focusing on tasks is claimed to increase system fragmentation. Well-designed processes make complexity manageable and reduce inefficiencies. BI can improve efficiency and agility in managing information for decision-making and has repeatedly been shown to deliver value in healthcare, including through process efficiency, reduced costs and improved patient care (Kao et al. 2016). Applying BI within a complex systems approach can support a better understanding of complex processes and their relationships, and avoids focusing on isolated tasks.

Real-time applications in healthcare have been used in health monitoring systems using physiological sensors on patients to enhance quality of life and reduce healthcare costs (Simpao et al. 2014; Wan et al. 2013); clinical decision-support (Mane 2012), and dashboards for operational performance monitoring and compliance (Simpao et al. 2014; Weiner et al. 2016). It is increasingly necessary for healthcare organizations to improve performance by creating a data-driven decision-making culture. Weiner et al. (2016) reported on the development of a real-time operational dashboard supporting data integration from multiple systems that was made available to all hospital staff with permissions, to create a culture of 
transparency and accountability. This required clinical and IT collaboration, and aligned organizational and unit level performance, supporting agility.

Making selected real-time information available to patients can support sensemaking, that is, making sense of the complexities of the decision by constructing a mental model. The mental model is a form of knowledge that clarifies the interrelationships between key factors involved in a problem (Wieke 1985). This has been shown to improve comprehension of events and support predictions about outcomes (Bagdasarov et al. 2016). Ultimately the decision to attend a particular emergency care service lies with the patient, and factors such as perceptions of their clinical condition, wait-time tolerance, travel mode and distance involved, past experiences and perceptions of available care available will all contribute to the final decision. The user plays an important role in the creation of the supply chain and BI decisionsupport aims to support the system by making real-time data available to patients, enabling them, in this case, to make an informed choice on treatment options and potentially changing ED attendance behavior.

\section{PROBLEM DESCRIPTION - OVERCROWDING OF ED IN T\&SD}

TSDFT operates one acute/ED hospital at Torbay Hospital and currently several community hospitals at Brixham, Dawlish, Newton Abbot, Paignton and Totnes. The community hospitals have MIUs that are equipped to provide services for minor conditions such as broken bones, muscle injury, minor head injury, cuts and wounds (dressing), minor burns, skin infections, etc. However, many potential users are unaware of the availability of these centers which may be appropriate to meet their needs close to where they are located. Therefore they usually choose to go to ED as they are confident they will be seen and have their medical needs met. This has led to overcrowding with the rate of ED attendance at Torbay hospital now above the national average with 39,419/100,000 population, compared with 30,041/100,000 in England (Turner, 2015). Another factor contributing to the overcrowding is the increased demand from tourists during the summer (by one estimate the catchment population of the trust increases by over 100,000 during this period).

Figure 1 presents a stacked histogram of the proportion of patients that were treated in the MIUs and Torbay ED. The source of the data is the healthcare information system that the Trust uses for recording urgent care data - Symphony ED (https://www.emishealth.com/products/symphony/); the time frame of analysis is $21 / \mathrm{July} / 2015$ to $29 / \mathrm{Jan} / 2016$. There are five time-intervals. Each interval consists of a lower and upper bound which represents the time it takes for a patient to be either seen, treated, admitted or discharged in under 4 hours of arriving in MIU/ED. For example, taking the case of time-frame '0-60 minutes', in Dartmouth MIU 94.44\% of the patients were either seen, treated, admitted or discharged within 0-60 minutes, for Teignmouth this was approx. $75 \%$, followed by Dawlish and Totnes at $73 \%$ and $71 \%$ respectively. On the other end of the scale are the 4-hour breaches $(240+$ minutes). In Dartmouth MIU there was no breach; Newton Abbot had a breach \% of only 0.04 , followed by Brixham at $0.21 \%$. As can be seen from Figure 1, the ED at Torbay hospital had the maximum number of breaches - one in four patients had to wait for more than 4 hours to be either seen, treated, admitted or discharged. Our analysis has shown that $66 \%$ of the overall number of patient attendance during this period was at Torbay. This compares to only 16\% for Newton Abbot MIU and 5.5\% for Dawlish. The remaining MIUs each treated less than $3 \%$ of the total number of patients requiring emergency attendance in T\&SD.

Our analysis shows that Torbay hospital not only has the highest number of 4-hour breaches (25.08\%) but, since it accounts for over $66 \%$ of patients being treated in the TSDFT urgent care network, this equates to approx. 9500 patients. Compared to this is the Newton Abbot MIU which has a breach percentage of less than $0.05 \%$ but still handles almost $16 \%$ of the emergency admissions. We therefore ask the question, can some of the demand at Torbay ED be redistributed to other large MIUs (like Newton Abbot)? Can a BI application help in lowering the overcrowding of ED by making available real-time $\mathrm{MIU} / \mathrm{ED}$ wait time information and whether this could influence/nudge the ED attendance behavior of the intended user of the urgent care system? 


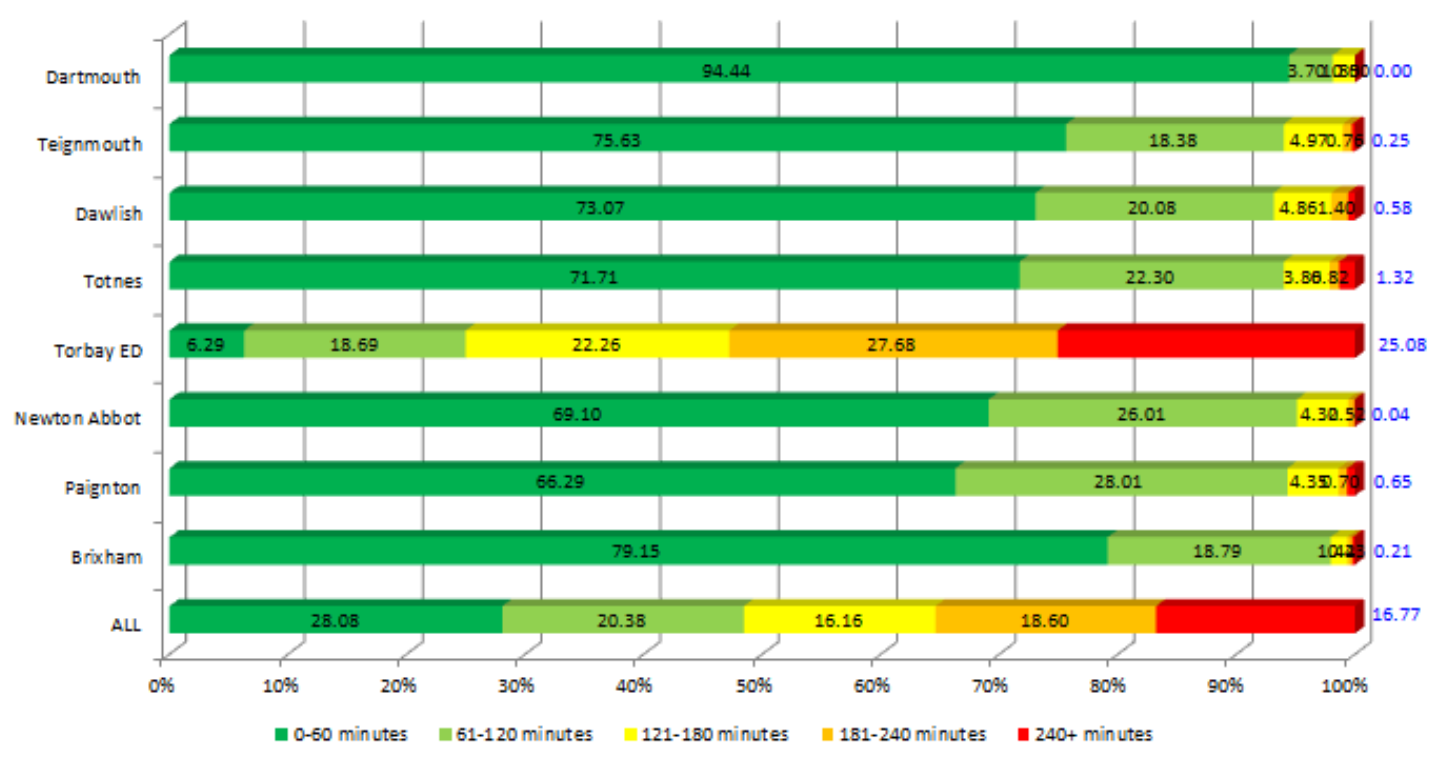

Figure 1: Proportion of patients being seen, treated, admitted or discharged within a certain time-interval (X-axis) at MIUs and Torbay ED (y-axis). Analysis of Symphony dataset - 21/07/2015 to 29/01/2016.

\section{BI APPLICATION ON MIU/ED WAIT TIME}

In a healthcare setting, the availability of real-time data empowers users and may increase their levels of self-activation in early reduction in anxiety. Our approach was therefore to enable those requiring medical attention to quickly identify MIUs that meet their needs. This was achieved by developing a web-based BI application that presented real-time data from the ED and the MIUs on current waiting time, the number of patients waiting to be seen and total number of patients in the department (Figure 2). This allowed those in need of urgent care to take an informed decision on whether their condition required attention from ED (which may have long waiting time) or they are better off receiving treatment at an MIU with a shorter wait time; the MIU could also be geographically closer to their current location. Further, this information allowed users to conceptualize decision making scenarios, for example, the trade-off involved in travelling to an MIU that is further away from home but with relatively less number of patients or be seen at the nearest MIU. The wider dissemination (and the resultant adoption) of the BI application has the potential to reduce some of the peaks in ED demand in T\&SD and make it easier to manage patient flow.

The BI application has been designed to benefit both internal and external actors (refer to section 1 for description of actors):

- External actor - Those who can be classed as worried well, parents with young children, young and middle aged adults and those unwilling to wait. For example, within T\&SD, the largest \% shift of attendees to ED is the 0-5 age group (9\%) and the 30-34 bracket (7\%), followed by the 40-49 age bracket (6\%) (Urgent Care Strategy, SD\&T CCG 2015).

- Internal actor - Health and care professionals (approx. 6,000 in T\&SD) who presently do not have mobile access to information about local health and care services, waiting times or where to direct people.

In relation to the external actors, by the very nature of the core purpose the application serves (urgent and emergency care), it is expected to be used infrequently by the user. However, we anticipate that its use will serve to better redistribute demand across the urgent care network and through this the NHS Trusts will ultimately benefit in the form of increased patient satisfaction, freeing up ED resources, achieving better staff/patient utilization rates at MIUs, and meeting the ED/MIU 4-hour target. The health and care 
professionals with TSDFT, on the other hand, are expected to use the application on a daily basis since it will assist them in providing better direction to patients to alternative services.

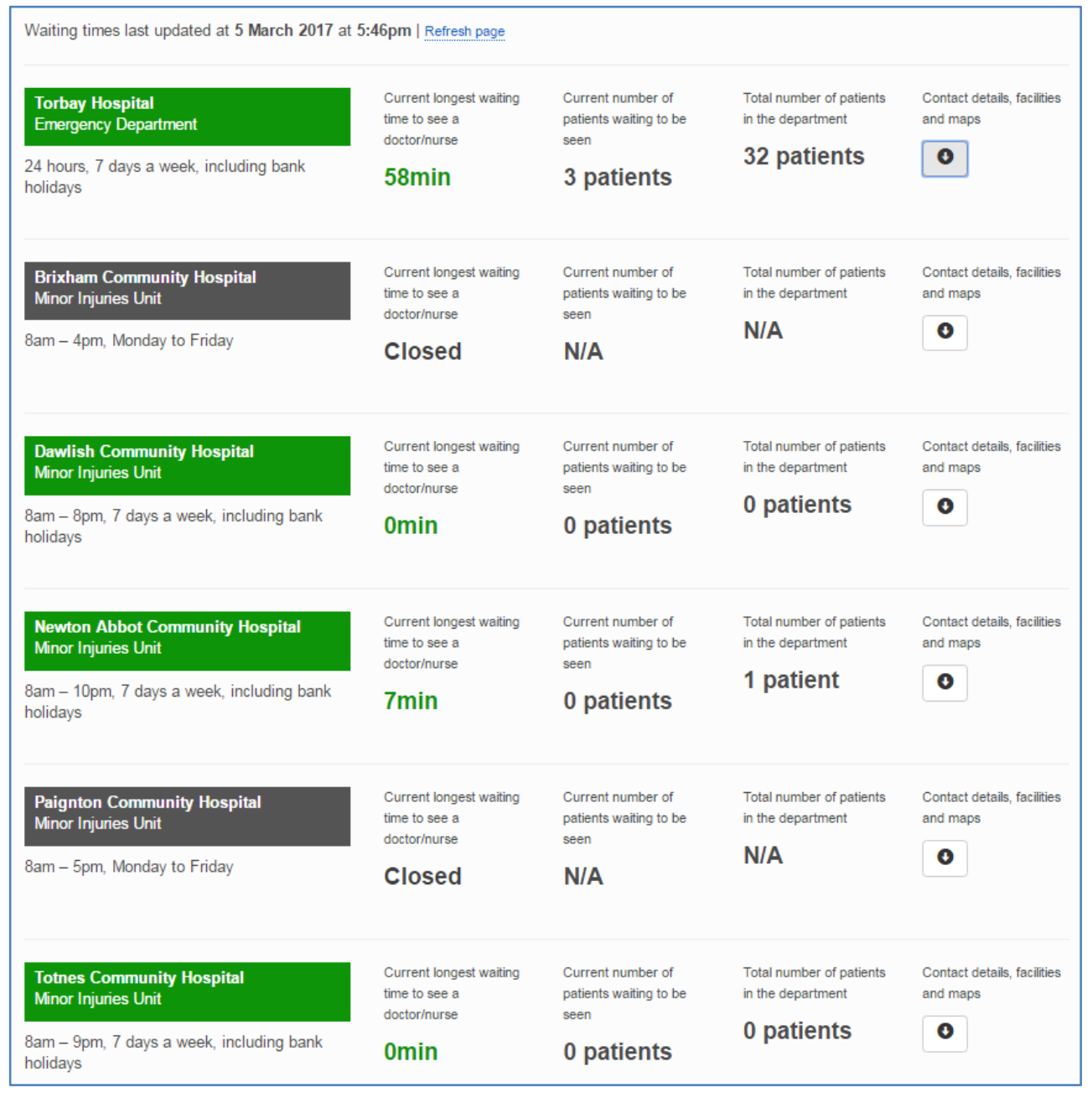

Figure 2: Live ED/MIU wait time information (TSDFT, 2016)

\section{ANALYSIS OF WEB TRAFFIC}

One measure of success is the number of users accessing the specific URL hosting the ED wait time BI application, and how it compares with the total traffic received by the TSDFT website. We have monitored this since the launch of the application in March 2016. For reference, the official TSDFT website is http://www.torbayandsouthdevon.nhs.uk/; the ED wait time application is hosted in the following URL: http://www.torbayandsouthdevon.nhs.uk/services/urgent-and-emergency-care/ed-miuwaiting-times/ (last accessed $5^{\text {th }}$ March 2017). The Trust uses Google Analytics, a free web analytics service offered by Google, to monitor and report on web traffic. Our period of analysis is $1^{\text {st }}$ March 2016 to $27^{\text {th }}$ September 2016 - a period of seven months.

During the aforementioned period, the number of page views related to the Trusts' website was over 630,000; of these, unique page views were approx. 472,000. How does this compare with the traffic which specifically accessed the BI application? Google Analytics reports that during this timeframe the application received more than 47,500 hits (equates to around $7.55 \%$ of the total traffic), of which around 33,000 were unique page hits (this is approx. $7.10 \%$ of the total unique hits received by the Trust website). When the application was first made live it received close to 15,000 hits, this is significantly more than the following months where we see that the web traffic has stabilized to approx. 4,000-6,000 hits. This is to be expected as any new information/innovation in the Trust is disseminated through 
multiple channels, and this would have contributed to the significantly more number of hits during the BI application launch. Our analysis of new versus returning users has shown that, of the total of nearly 47,500 hits since its launch, new visitors have accessed the site over 14,000 times (approx. 30\%) with the remaining $70 \%$ hits originating from returning visitors. These latter are most likely the internal actors (the heath and care professionals working at the Trust).

\section{FUTURE WORK AND CONCLUSION}

Creating a decision-making workflow with multiple stakeholders in a live running complex system with incomplete domain knowledge and fragmented data acquisition is a challenge that many legacy systems are faced with. This is true also for healthcare. Operational decisions have traditionally been considered to be an internal function of the hospital and stakeholders like the clinical and the operational staff (managers). In this study we considered one additional stakeholder group, namely, the intended users of the system (the patients) and how complex decision making may benefit from the end users' treatment choices. The aim of this research was to provide users with real-time information on local services that can effectively assess and treat minor ailments, thereby reducing the number of avoidable attendances at ED by diverting patients away from ED to local services; furthermore, to investigate whether such a technological solution could help bring change in people's behavior. To this end we implemented a BI application which integrates data feeds on waiting times from ED and Minor Injury Units. It is expected that this will empower users by enabling them to make an informed choice on treatment options; this conceivably reduces patient anxiety and changes ED attendance behavior.

At the start of the project we identified the following three outcomes as our measure of success: (a) The BI application will access real-time ED and MIU data on waiting times which was held by TSDFT. This has been realized through 'NHS Data Model and Dictionary for England' and the 'CDS V6-2 Type 010 Accident and Emergency Commissioning Data Set' (http://www.datadictionary.nhs.uk/) which carries the data for an A\&E attendance. (b) Non-trivial number of user access the BI application; both new and returning users. As can be seen from the data on number of website hits to this specific URL (see section 5), we are on target to realize this measure. (c) The BI intervention has had a measurable impact in the reduction of overcrowding in ED and diverting non-emergency attendance from ED to MIUs. In other words, the technology intervention had contributed in changing/nudging the ED attendance behavior of the intended users of the system. In realization of this intended outcome, we are currently developing an app which would enable us to not only deliver the live wait times through a mobile, but would report waiting time and travel time based on users' location (using GPS). This is work in progress and we hope to present the results in a future publication.

Our BI application can be implemented in other NHS Trusts in England since the real-time data on waiting times, that are held by foundation and acute hospital trusts, are based on the NHS data model and the 'Accident and Emergency Commissioning Data Set' (HSCIC, 2016). In other words, this is the single view of data and all databases that carry the data for ED attendance must confirm to it. This makes for easier implementation of the technological solution.

\section{Acknowledgement}

We would like to acknowledge the ESRC Impact Acceleration Account on Project Co-creation for the project on "Systems Modelling and Computer Simulation of Urgent and Emergency Care in Torbay \& South Devon". We acknowledge Prof. Ben Benjamin (Consultant Acute Physician), Stephen Judd (Informatics Manager) and Stephen Macey (Performance Information Analyst), Liz Procter (Informatics Manager - Transformation Team), Paul Hurrell (Head of Innovation, Torbay \& South Devon CCG) from TSDFT; Pam Prior (Chair of the Healthwatch Torbay), Nicky Morrish (Service Development Manager and DOS for Devon \& Cornwall \& IOS), Julie Eatock (Brunel University) and My Oxygen.

\section{REFERENCES}


Bagdasarov, Z., Johnson, J.F., MacDougall, A.E., Logan, M.S., Connelly, S. and Mumford, M.D. 2016. "Mental models and ethical decision making: The mediating role of sensemaking. Journal of Business Ethics. Vol. 138(1). pp. 133-144

Daellenbach H. G., McNickle, D.C. and Dye, S. 2012. "Management Science: Decision-Making Through Systems Thinking”. New York: Palgrave Macmillan

Evelson, B. 2010. “The Forrester Wave ${ }^{\mathrm{TM}}$ : Enterprise Business Intelligence Platforms”. Forrester Research Inc. www.forrester.com/go? objectid=RES56280. Accessed 20th January 2013.

HSCIC. 2016. "NHS Data Model and Dictionary for England - CDS V6-2 Type 010 - Accident and Emergency Commissioning Data Set”. http://www.datadictionary.nhs.uk. Last accessed July 2016.

Ivanov, D., Sokolov, B. and Kaeschel. 2010. "A Multi-structural framework for adaptive supply chaing planning and control operations with structure dynamic considerations." European Journal of Operational Research. Vol 200(2). pp.409-420

Kao, H., Yu, M., Masud, M., Wu, W, Chen, L. and Wu, Y. 2016. Design and evaluation of hospital based business intelligence (HBIS): A foundation for design science research methodology. Computers in Human Behaviour. Vol. 62: 495-505

Kluth, A., Jager, J., Schatz, A. and Bauernhansl, T. 2014. "Evaluation of Complexity Management Systems - Systematical and maturity-based approach." Procedia CIRP. Vol. 17. pp. 224-229

Mane, K.K., Bizon, C., Schmitt, C., Owen, P., Burchett, B., Pietrobon, R. and Gersing, K. 2012. "VisualDecisionLinc: A visual analytics approach for comparative effectiveness-based clinical decision support in psychiatry". Journal of Biomedical Informatics. Vol. 45(1). pp. 101-106

May, M.C. 2006. "A rational model for assessing and evaluating complex interventions in healthcare". BMC Health Services Research. Vol. 6. pp. 86-96

Mettler, T. and Vimarlund, V. 2009. "Understanding business intelligence in the context of healthcare". Health Informatics Journal. Vol.15(3). pp. 254-264.

Ngai, E.W.T., Peng, S., Alexander, P. \& Moon, K. 2014. "Decision support and intelligent systems in the textile and apparel supply chain: An academic review of research articles". Expert Systems with Applications. Vol.41(1). pp.81-91

NHS England. 2015. "Five Year Forward Plan". https://www.england.nhs.uk/wpcontent/uploads/2014/10/5yfv-web.pdf Last accessed July 2016.

Paley, J. and Eva, G. 2011. "Complexity theory as an approach to explanation in healthcare: a critical discussion". International Journal of Nursing Studies. Vol.48(2). pp.269-79

Shadi, S.A., and Mustafee, N. 2013. "Business intelligence in NHS Wales". In Proceedings of the 2013 UK Academy for Information Systems International Conference (UKAIS2013), March 18-20, 2013, Worcester College, University of Oxford, Oxford, UK.

South Devon \& Torbay CCG. 2015. "Urgent care strategy: 2014/15 - 2019/20" South Devon and Torbay Clinical Commissioning Group.

Simpao, A., Ahumada, L., Galvez, J. and Rehman, M.A. 2014. "A review of analytics and clinical informatics in health care". Journal of Medical Systems. Vol. 38(4). pp. 38-45

Spruit, M., Vroon, R. and Batenburg, R. 2014. "Towards healthcare business intelligence in long-term care: An explorative case study in the Netherlands". Computers in Human Behaviour. Vol.30. pp. 698707

TSDFT. 2016. "MIU waiting times". http://www.torbayandsouthdevon.nhs.uk/services/urgent-andemergency-careled-miu-waiting-times/\#brixham-community-hospital Last accessed March 2017. 
Turner, J. 2015. "Managing Avoidable Demand for an Emergency Department - A research project for South Devon Healthcare Foundation Trust".

Urgent and Emergency Care Review Team. 2013. "Transforming urgent and emergency care services in England Urgent and Emergency Care Review". http://www.nhs.uk/NHSEngland/keoghreview/Documents/UECR.Ph1Report.FV.pdf Last accessed July 2016

Walker, J.M. and Carayon, P. 2009. "From tasks to processes: the case for changing health information technology to improve health care". Health Affairs. Vol. 28(2). pp.467-77

Wan, J., Zou, C., Ullah, S., Lai, C., Zhou, M. and Wang, X. 2013. "Cloud-enabled wireless body area networks for pervasive healthcare”. IEEE Network Magazine. Vol. 27(5). pp. 55-61

Weiner, J., Tanniru, J. \& Khuntia, J., Bobryk, D., Naik, M., Lepage, K. 2016. "Digital leadership in action in a hospital through a real time dashboard system implementation and experience."

Wieke, K.E. 1995. "Sensemaking in Organizations". Thousand Oaks, CA: Sage.

Yurtseven, M.K. and Buchanan, W.W. 2016. "Complexity decision making and general systems theory: An educational perspective. Sociology Study. Sociology Study. Vol.6(2): pp. 77-9

\section{AUTHOR BIOGRAPHIES}

NAVONIL MUSTAFEE is Senior Lecturer in Operations and Supply Chain Management at University of Exeter Business School. His research interests are in simulation methodologies, parallel and distributed simulation and healthcare simulation. His e-mail address is n.mustafee@exeter.ac.uk and his web page is http://sites.google.com/site/navonilmustafee/.

JOHN POWELL is Professor in Strategy at University of Exeter Business School.. His research interests are in systems modelling of strategic situations, scenario planning and modelling, knowledge in strategy, knowledge management. His email address is j.h..powell@exeter.ac.uk.

SUSAN MARTIN is Associate Director, Quality Improvement, Torbay and South Devon NHS Foundation Trust. Her email is susan.martin@nhs.net.

ANDREW FORDYCE is Consultant Oral \& Maxillofacial Surgeon and Clinical Systems Engineer, Torbay Hospital, Torbay and South Devon NHS Foundation Trust. His email address is andrew.fordyce@nhs.net.

ALISON HARPER is a Post Graduate Research (PGR) research student at University of Exeter Business School. Her research interests are in healthcare simulation and analytics. Her e-mail address is ah596@exeter.ac.uk. 